Original Contribution

\title{
The role of p38 signaling and poly(ADP-ribosyl)ation-induced metabolic collapse in the osteogenic differentiation-coupled cell death pathway
}

\author{
Agnieszka Robaszkiewicz ${ }^{\mathrm{a}, \mathrm{b}}$, Zsuzsanna Valkó ${ }^{\mathrm{a}}$, Katalin Kovács ${ }^{\mathrm{a}, \mathrm{c}}$, Csaba Hegedűs ${ }^{\mathrm{a}}$, \\ Edina Bakondi ${ }^{a}$, Péter Bai ${ }^{\mathrm{a}, \mathrm{c}, \mathrm{d}, \mathrm{e}}$, László Virág ${ }^{\mathrm{a}, \mathrm{c}, *}$ \\ a Department of Medical Chemistry, Medical and Health Science Center, University of Debrecen, Debrecen, Hungary \\ ${ }^{\mathrm{b}}$ Department of Environmental Pollution Biophysics, Faculty of Biology and Environmental Protection, University of Lodz, Poland \\ ${ }^{\mathrm{c}}$ MTA-DE Cell Biology and Signaling Research Group, Debrecen, Hungary \\ d Research Center for Molecular Medicine, University of Debrecen, Debrecen, Hungary \\ e MTA-DE Lendület Laboratory of Cellular Metabolism Research Group, Debrecen, Hungary
}

\section{A R T I C L E I N F O}

Article history:

Received 14 May 2014

Received in revised form

11 July 2014

Accepted 18 July 2014

\section{Keywords:}

Osteogenic differentiation

Hydrogen peroxide

Poly(ADP-ribosyl)ation

PARP1

Cell death

p38 MAP kinase

\begin{abstract}
A B S T R A C T
Osteogenic differentiation is a multistep process regulated by a diverse set of morphogenic and transcription factors. Previously we identified endogenous hydrogen peroxide-induced poly(ADP-ribose) polymerase-1 (PARP1) activation as a mediator of osteodifferentiation and associated cell death. Here we set out to investigate whether or not activation of PARP1 is dependent on DNA breaks and how PARP1 mediates cell death during osteodifferentiation of mesenchymal stem cells and SAOS-2 cells. Here we show that the MAP kinases $\mathrm{p} 38$, JNK, and ERK1/2 become activated during the differentiation process. However, only p 38 activation depended both on hydrogen peroxide production and on PARP1 activation as the hydrogen peroxide decomposing enzyme catalase, the PARP inhibitor PJ34, and the silencing of PARP1 suppressed p38 activation. Inhibition of p38 suppressed cell death and inhibited osteogenic differentiation (calcium deposition, alkaline phosphatase activity, and marker gene expression) providing further support for the close coupling of osteodifferentiation and cell death. Metabolic collapse appears to be central in the hydrogen peroxide-PARP1-p38 pathway as silencing PARP1 or inhibition of p38 prevented differentiation-associated loss of cellular NAD, inhibition of mitochondrial respiration, and glycolytic activity. We also provide evidence that endogenous hydrogen peroxide produced by the differentiating cells is sufficient to cause detectable DNA breakage. Moreover, p38 translocates from the cytoplasm to the nucleus where it interacts and colocalizes with PARP1 as detected by immunoprecipitation and immunofluorescence, respectively. In summary, hydrogen peroxide-induced PARP1 activation leads to p38 activation and this pathway is required both for the successful completion of the differentiation process and for the associated cell death.
\end{abstract}

(c) 2014 Elsevier Inc. All rights reserved.
Abbreviations: AIF, apoptosis inducing factor; AP-1, activating protein-1; AP-2, activating protein 2; BMP2, bone morphogenetic protein 2; cMSC, chorion-derived mesenchymal stem cells; CTRL, control cells; DIFF, differentiating cells; ECAR, extracellular acidification rate; FGF, fibroblast growth factor; GAPDH, glyceraldehyde 3-phosphate dehydrogenase; MKP-1, mitogen-activated protein kinase (MAPK) phosphatase 1; NF-кB, nuclear factor kappa B; OCR, oxygen consumption rate; OD, optical density; PAR, poly(ADP-ribose); PARG, poly(ADP-ribose) glycohydrolase; PARP1, poly(ADP-ribose) polymerase-1; PARylation, poly(ADP-ribosyl)ation; Runx2, runt-related transcription factor 2; SEM, standard error of the mean; SIRT1, sirtuin 1; TEF1/Max, transcription enhancer factor 1/Max; TGF, transforming growth factor; z-VAD-fmk, benzyloxycarbonyl-Val-Ala-Asp (OMe)

fluoromethylketone

* Corresponding author at: Department of Medical Chemistry, Faculty of Medicine, University of Debrecen, Nagyerdei krt 98, H-4032 Debrecen, Hungary. Fax: + 3652412566

E-mail address: lvirag@med.unideb.hu (L. Virág).

\section{Introduction}

Membranous ossification involves the differentiation of mesenchymal stem cells into osteoprogenitors and eventually osteoblasts. Differentiated osteoblasts produce extracellular matrix with Type I collagen considered as the prototypical component of the osteoid matrix [1]. In turn, mineralization (deposition of calcium, magnesium, and phosphate containing hydroxyapatite) completes the process resulting in a bone tissue that is hard and strong enough to fulfill skeletal function [2]. This complex series of events is regulated by a diverse set of transcription factors such as osterix, Runx2, Groucho/TLE, or Twist and soluble morphogenic factors such as BMPs, TGF $\beta$, and Wnt [3-6]. 
Osteogenic differentiation is accompanied by massive cell death with the majority of differentiating cells undergoing apoptosis [6,7]. The identity and regulation of the cell death subroutine mediating osteogenic differentiation-coupled cell death, however, have not yet been completely characterized. Production of reactive oxygen species has been shown to accompany osteogenesis [8,9] and our group has established the contribution of the hydrogen peroxide-induced poly(ADP-ribose) polymerase-1 (PARP1) pathway to osteogenic differentiation and related cell death [7]. Poly (ADP-ribosyl)ation is a protein modification catalyzed by some members of the PARP enzyme family. PARP1 is a DNA nick sensor enzyme catalyzing cleavage of NAD to nicotinamide and ADPribose and covalently attaching the latter to suitable acceptor proteins. In turn, the first protein-bound ADP-ribose unit can serve as new site for the addition of further ADP-ribose moieties resulting in the formation of poly(ADP-ribose) (PAR) $[10,11]$. The wide range of biological activities of PARP enzymes ranging from regulation of genome organization, transcription, telomere length, and metabolism to facilitation of DNA repair and propagation of cell death are due to (a) covalent PARylation of target proteins, (b) noncovalent binding of free or protein-bound PAR polymers to proteins, (c) depletion of cellular NAD and ATP pools, (d) physical interaction of PARPs with other proteins [12-16]. The role of PARP1 in cell death regulation is complex and largely depends on the cellular model and the activating stimulus. Mechanisms underlying PARP1-mediated necroptotic cell death include cellular metabolic insufficiency (due to NAD and ATP depletion), PARinduced nuclear translocation of AIF (apoptosis inducing factor), and interference with prosurvival and prodeath signaling pathways [11,17-19]. As for the latter, signaling routes mediated by MAP kinases have been shown to be intertwined with PARylation [20-23]. Of note, it has been shown that the expression of MAP kinase phosphatase 1 (MKP-1) is negatively regulated by PARP1 strengthening prodeath pathways mediated by JNK and p38 MAP kinases [24]. Which of these three major PARP1-dependent mechanisms are responsible for the cell death of differentiating osteoprogenitors and whether they are connected to the hydrogen peroxide-mediated redox regulatory pathway that we have previously identified remain to be determined.

Since we have previously demonstrated the lack of AIF translocation during osteogenic differentiation-coupled cell death [7], here we set out to investigate the role of MAP kinase signaling and compromised cellular energetics as possible mediators of PARP1dependent cell death during osteogenic differentiation. We show that while all three MAP kinases become activated during the course of osteogenic differentiation, inhibition or silencing of PARP1 only suppresses the activation of $\mathrm{p} 38$, while Erk1/2 activation was augmented and JNK activation was not affected. Moreover, p38 is a downstream mediator of the hydrogen peroxidePARP1 cytotoxic pathway mediating not only cell death but the osteogenic differentiation process as well.

\section{Materials and methods}

Bis-benzimide trihydrochloride (Hoechst H33342), 1,4-diazabicyclo[2.2.2] octane (DABCO), Mowiol 4-88, protease inhibitor cocktail (PIC), dexamethasone, $\beta$-glycerophosphate disodium salt hydrate, 2-phospho-L-ascorbic acid trisodium salt, vitamin $\mathrm{D}_{3}$ solution, catalase from bovine liver, nitrocellulose membrane, 3,3'-diaminobenzidine (DAB), SB239063 and SB203580 (p38 inhibitors), SP600125 (JNK inhibitor), glycylglycine, niacinamide, alkohol dehydrogenase from Saccharomyces cerevisiae, (3-(4,5-dimethylthiazol-2-yl)-2,5-diphenyltetrazolium bromide (MTT), phenazine methosulfate, nicotinamide adenine dinucleotide (NAD), PJ34, lentiviral silencing vectors, antimycin A, oligomycin, and carbonyl cyanide 4-(trifluoromethoxy) phenylhydrazone (FCCP) were purchased from Sigma Aldrich (Budapest, Hungary). Dulbecco's modified Eagle medium with sodium pyruvate and low glucose (DMEM), penicillin-streptomycin solution, and fetal bovine serum were from Life Technologies (Budapest, Hungary). The SuperSignal West Pico Chemiluminescent Substrate, Pierce BCA Protein Assay kit was purchased from Thermo Scientific (Waltham, MA). U0126 (MEK1/2 inhibitor), MAPK, and phosphoMAPK Family Antibody Sampler Kits were from Cell Signaling Technology (Budapest, Hungary). The FITC Annexin V Apoptosis Detection Kit I was purchased from BD Biosciences (Pécs, Hungary). XF96 FluxPak and XF Assay Medium were from Seahorse Biosciences (North Billerica, MA, USA).

\section{Isolation and phenotyping of mesenchymal stem cells}

Chorion-derived mesenchymal stem cells (MSC) were isolated from human placentas (as approved by the regional research ethics committee under license number DEOEC-RKEB-29462009) and phenotyped on the basis of surface antigen pattern using cell staining with appropriate antibodies combined with flow cytometric analysis (Becton Dickinson BD FACSCalibur and BD Multiset Software v3.0x for Mac OS X) as described previously [7]. Only cells positive for CD73, CD90, CD105, and CD166 and negative for CD34, CD45, vWF, and HLA-G were used in the experiments.

\section{Cell culture and differentiation}

SAOS-2 human osteosarcoma cells and chorion-derived mesenchymal stem cells were cultured and differentiated as previously desribed [7].

\section{Generation of SAOS-2 cell line with stably silenced PARP1}

PARP1 silenced (shPARP1) SAOS-2 cells were obtained by transduction with lentiviral vectors containing the insert [constructs TRCN0000007929] as described previously [25], while cells transduced with pLKO.1-puro empty vector served as a negative control (CTRL). Transduced cells were cultured and differentiated in the presence of puromycin $(5 \mu \mathrm{g} / \mathrm{ml})$. Stability of silencing was checked regularly by detection of PARP1 mRNA and protein levels (qPCR and Western blot, respectively).

\section{Comet assay}

In order to study ROS-induced DNA damage, cells were collected on different days of differentiation and were subjected to the comet assay procedure as previously described [26]. Images were acquired with a Zeiss Axiolab microscope (Carl Zeiss, Oberkochen, Germany) with a Zeiss Axiocam digital camera. The degree of DNA damage was estimated on the basis of tail DNA (\%) calculated with CASPLab Comet Assay Software. Red to black ratio of acquired images was enhanced by adjustment of brightness, contrast, hue, and saturation. Subsequent conversion of color images to grayscale was followed by inversion. These adjustments were carried out on all images in an identical manner.

\section{Immunoprecipitation}

Immunoprecipitation of PARP1, p38, or phosphorylated p38 (P-p38) was carried out according to Rothgiesser et al. [27]. Cell lysates were incubated with either anti-PARP1 rabbit polyclonal antibody [generated against a peptide fragment (amino acids 506523) of human PARP1] or anti-p38 or anti-phospho-p38 antibodies. Protein-antibody complexes were pulled down with Dynabeads Protein G and subjected to Western blot analysis [7]. Images were acquired using a FluorChem FC2 AIC imager. 


\section{Western blot}

In order to determine the involvement of MAP kinases in osteogenic differentiation, analysis of MAP kinase phosphorylation was carried out with Western blot as previously described [7].

\section{Cell viability}

The percentage of viable and nonviable cells was quantified using an Annexin V-FITC/propidium iodide kit (Becton-Dickinson) as described previously [7]. Cells were analyzed with a BectonDickinson BD FACS Calibur flow cytometer and the acquired data were processed with BD Multiset software version 3.0x for Mac OS X (Becton-Dickinson). Cells were classified as "viable" (weak green and weak red fluorescence), "apoptotic" (strong green and weak or strong red fluorescence), and "necrotic" (weak green and strong red fluorescence).

\section{Characterization of osteogenic differentiation}

Osteogenic differentiation was characterized by measuring calcium deposition (Alizarin red staining), quantifying alkaline phosphatase activity (enzyme activity assay), and the expression of osteogenic marker genes (osterix, RUNX2 and BMP-2) by qPCR. All these assays were carried out exactly as described in Robaszkiewicz et al. [7]. The list of primers used is given in Supplementary Material 1.

\section{RNA extraction, reverse transcription, and real-time PCR analysis}

Total RNA was isolated from cells with TRI reagent as described previously [7]. Isolated RNA was reverse-transcribed to cDNA with the High Capacity cDNA reverse transcription kit purchased from Fermentas, Thermo Scientific (Waltham, MA). For real-time PCR Maxima SYBR Green qPCR Master Mix (Fermentas, Thermo Scientific, Waltham, MA) was used. Genes were amplified with an Applied Biosystems 7500 Fast Real-Time PCR system. The expression levels of genes analyzed were normalized to glyceraldehyde3-phosphate dehydrogenase as reference housekeeping gene.

\section{NAD assay}

The intracellular concentration of NAD was measured in differentiating cells with the recycling assay according to previously published protocol [25]. Data were normalized to protein determined with a Pierce BCA protein assay kit (according to instructions provided by the manufacturer).

\section{Metabolic activity}

Bioenergetic analysis was carried out using the Seahorse XF96 Analyzer (Seahorse Biosciences, MA, USA) as described in Modis et al. [28], which allows the simultaneous and real-time analysis of the oxygen consumption rate (OCR) and extracellular acidification rate (ECAR), which correspond to mitochondrial and glycolytic activity of cells, respectively. Prior to the analysis, differentiating medium was changed to unbuffered DMEM medium supplemented with $1 \%$ glucose. Sequential injection of oligomycin $(20 \mu \mathrm{g} / \mathrm{ml})$, FCCP $(9 \mu \mathrm{M})$, and antimycin $(20 \mu \mathrm{g} / \mathrm{ml})$ through Seahorse FluxPak cartridges permitted the determination of the following metabolic parameters: $\mathrm{ECAR}_{\mathrm{NM}}$, nonmitochondrial proton release, which represents glycolytic activity; $\mathrm{OCR}_{\mathrm{BR}}$, basal respiration, which corresponds to ATP-linked mitochondrial oxygen consumption, maximum respiratory rate, and respiratory reserve capacity $\left(O C R_{M R R}\right.$ and $O C R_{R R C}$, respectively) as well as nonmitochondrial oxygen consumption.
Immunocytochemistry and immunofluorescence

8-Hydroxydeoxyguanosine (8OHdG), a marker of oxidative DNA damage, was detected by immunocytochemistry. Preparation of samples and denaturation of cellular DNA was performed as described in Yamamoto et al. [29]. After 30 min incubation with 8OHdG antibody (Jaica, Shizuoka, Japan; clone N45.1; 1:30 dilution in $5 \% \mathrm{FBS}$, at $37^{\circ} \mathrm{C}$ ) a standard immunocytochemistry protocol was followed as previously described [25]. Samples were mounted in Mowiol and viewed with a Zeiss Axiovision microscope.

Colocalization of p38 and PARP1 was determined by immunofluorescent double staining using mouse anti-PARP1 (SigmaAldrich, Budapest, Hungary) and rabbit polyclonal anti-p38 antibodies (Cell Signaling Technology, Danvers, MA, USA) followed by incubation with AlexaFluor633-conjugated anti-mouse and AlexaFluor488-conjugated anti-rabbit IgG antibody (Life Technologies, Budapest, Hungary). Samples were viewed and imaged with a Leica SP8 confocal microscope.

\section{NADPH oxidase activity}

Activity of NADPH oxidase was measured according to Wang et al. [30]. The ratio of lucigenin oxidation was normalized to protein content (quantified with a Pierce BCA protein assay kit).

\section{Mitochondrial superoxide production}

Mitochondrial superoxide production was monitored on the basis of MitoSOX oxidation as described in Robaszkiewicz et al. [31]. Cells were analyzed with a Becton-Dickinson BD FACS Calibur flow cytometer and the acquired data were processed with BD Multiset software version 3.0x for Mac OS X (Becton-Dickinson).

\section{Results}

\section{Osteogenic differentiation is accompanied by DNA breakage}

Since PARP activation may be induced by DNA breaks and may also occur in the absence of DNA damage [16], first we set out to look for signs of DNA breaks in differentiating cultures. Differentiation of both MSC and SAOS-2 cells was accompanied by DNA strand breaks as detected with the comet assay (Fig. 1a). Of note, the appearance of DNA damage corresponded to the pattern of hydrogen peroxide release and PAR synthesis as documented previously [7]. Moreover, addition of the hydrogen peroxide decomposing enzyme catalase to the osteogenic differentiation medium prevented the formation of DNA strand breaks (Fig. 1a), indicating that hydrogen peroxide produced in the course of osteogenic differentiation is responsible for DNA breakage. The oxidative nature of DNA damage is further supported by the presence of $80 \mathrm{HdG}$ in the differentiating cells, as detected by immunocytochemistry (Fig. 1b).

Activation profiling of MAP kinases during osteogenic differentiation of SAOS-2 and mesenchymal stem cells

Enzymes belonging to the family of mitogen-activated protein kinases (MAPK) p38, JNK, and ERK1/2 play diverse roles in various models of cell death [32-35]. Osteogenic differentiation in SAOS-2 and MSC cells was also accompanied by marked changes in the phosphorylation state of three major MAP kinases (Fig. 2, Fig. S1, and Fig. S2). By day 6 of differentiation the phosphorylation level of p38 kinase increased approximately 7 fold in both cell types as compared to day 0 (Fig. 2). Similarly, phosphorylation of both ERK (Fig. S1) and JNK (Fig. S2) also changed significantly during the 
a
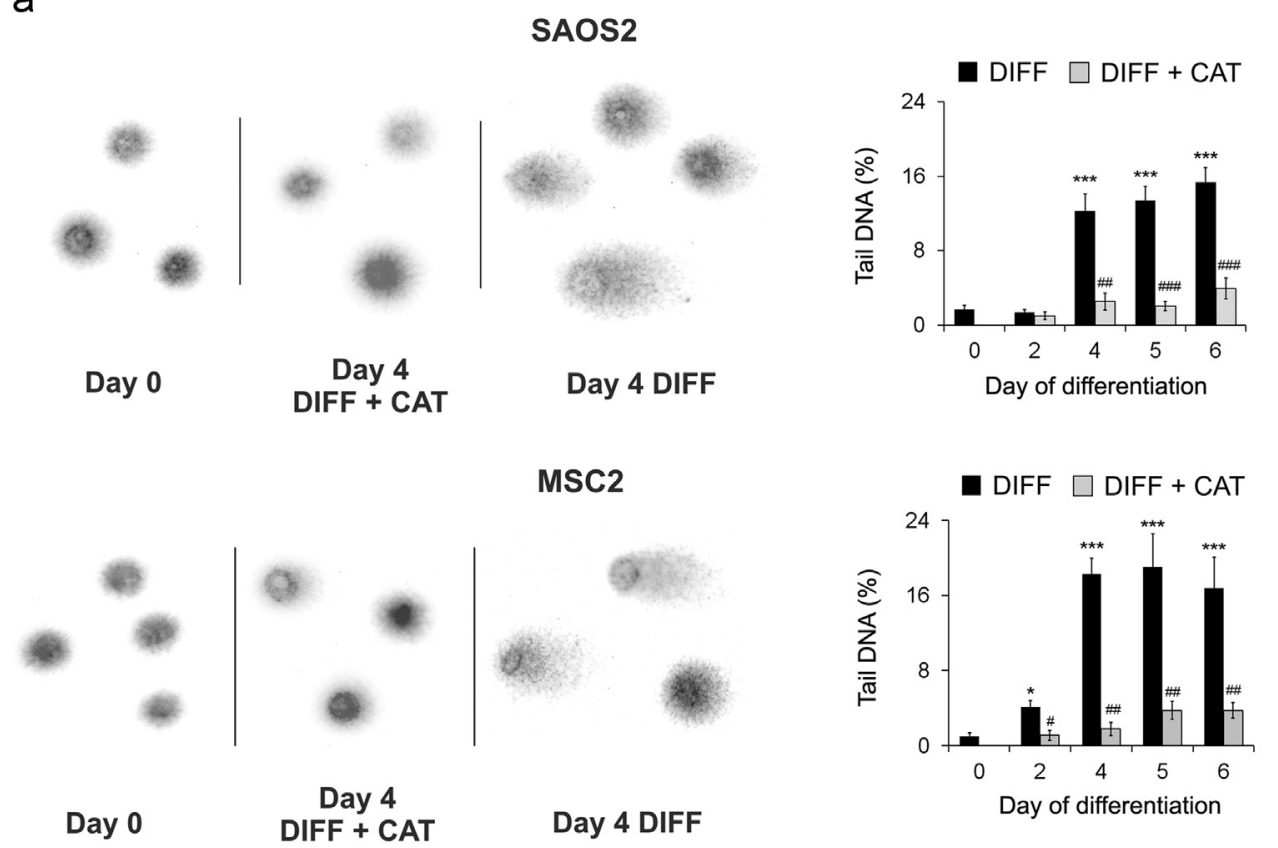

b

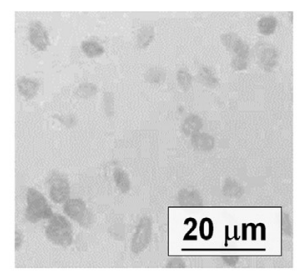

Day 0

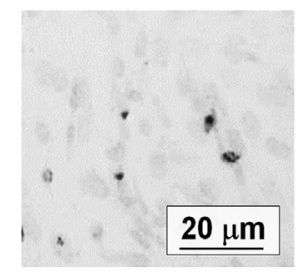

Day 3 DIFF

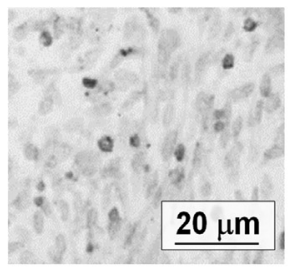

Day 6 DIFF

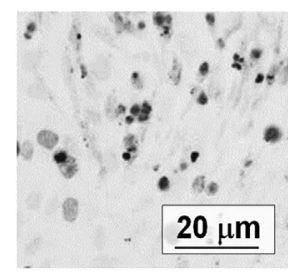

UV

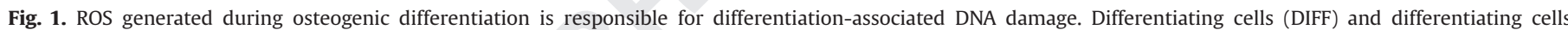

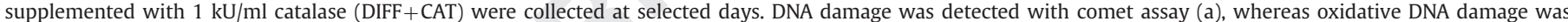

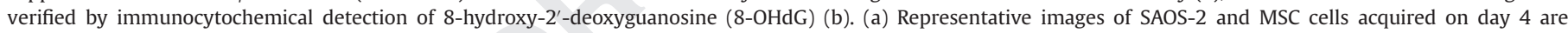

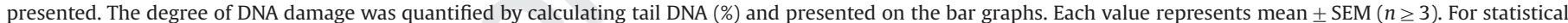

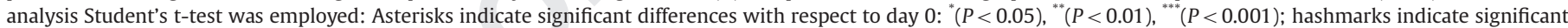

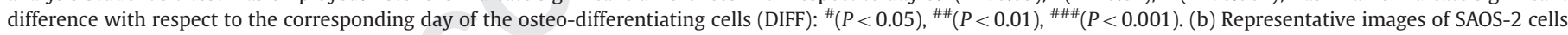
stained for 8OHdG and acquired on days 0,3 , and 6 are presented. UV irradiated SAOS-2 cells were used as positive control.

course of differentiation with some notable differences between SAOS-2 and MSC cells. Whereas in MSC an early activation of JNK and ERK1/2 (on days 1 and 2, respectively) could be observed, in SAOS-2 cells basal JNK phosphorylation was slightly downregulated in the first 2 days followed by a late (days 4 and 5) upregulation. Activation of ERK1/2 began to increase slowly on day 4 .

\section{The hydrogen peroxide-PARP1 pathway is intertwined with MAP kinase activation}

In order to confirm that the previously described hydrogen peroxide-PARP1 pathway is connected to the activation of one or more MAP kinases we have investigated the effects of catalase that decomposes hydrogen peroxide and the pharmacological PARP inhibitor PJ34. We found that catalase $(1 \mathrm{kU} / \mathrm{ml})$ prevented the phosphorylation of p38 kinase in both MSC and SAOS-2 cells (Fig. 2) but had no major effect on the ERK (Fig. S1) and JNK pathways (Fig. S2). The PARP1 inhibitor PJ34 $(1 \mu \mathrm{M})$ also suppressed p38 activation (Fig. 2) but augmented ERK phosphorylation (Fig. S1) without affecting JNK activation (Fig. S2). In order to prove that the primary target of the PARP inhibitor PJ34 is PARP1 in this model, we silenced the parp1 gene in SAOS-2 cells and performed MAP kinase profiling in these cells (shPARP1 cells).
Data obtained with shPARP1 cells phenocopied results obtained with the PARP inhibitor (Fig. 2, S1, and S2) indicating that PARP1 indeed inversely regulates $\mathrm{p} 38$ and ERK kinases during osteogenic differentiation. (Similar to the PARP inhibitor PJ34, PARP1 silencing had no effect on the activation of JNK.) As literature data suggested a possible cross-inactivation between p38 and ERK [36], we have investigated whether augmentation of ERK activation by PARP inhibition is due to suppression of $\mathrm{p} 38$. We could not confirm this hypothesis in our model of osteogenic differentiation as inhibition of p38 by the pharmacological inhibitor SB239063 $(5 \mu \mathrm{M})$ had no effect on the course of ERK activation either in SAOS-2 or in MSC cells (data not shown).

\section{p38 mediates osteogenic differentiation-coupled cell death}

Previously we have shown that hydrogen peroxide-induced PARP activation is required for osteogenic differentiation via mediating differentiation-coupled cell death [7]. Here we set out to investigate whether any of the MAP kinases are involved in this regulatory pathway. We found that inhibition of $\mathrm{p} 38$ but not that of ERK (MEK) or JNK protected cells from apoptotic death accompanying osteogenic differentiation (Fig. 3). In fact, pretreatment of cells with ERK or JNK inhibitors slightly sensitized cells to rather than protected cells from apoptosis. These data suggest that p38 is 
a

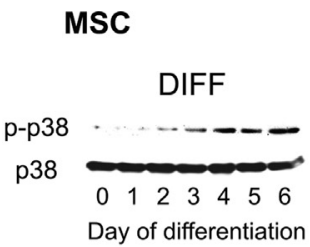

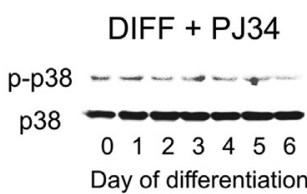

Day of differentiation

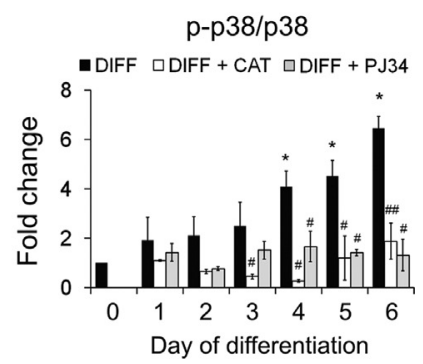

b

SAOS2
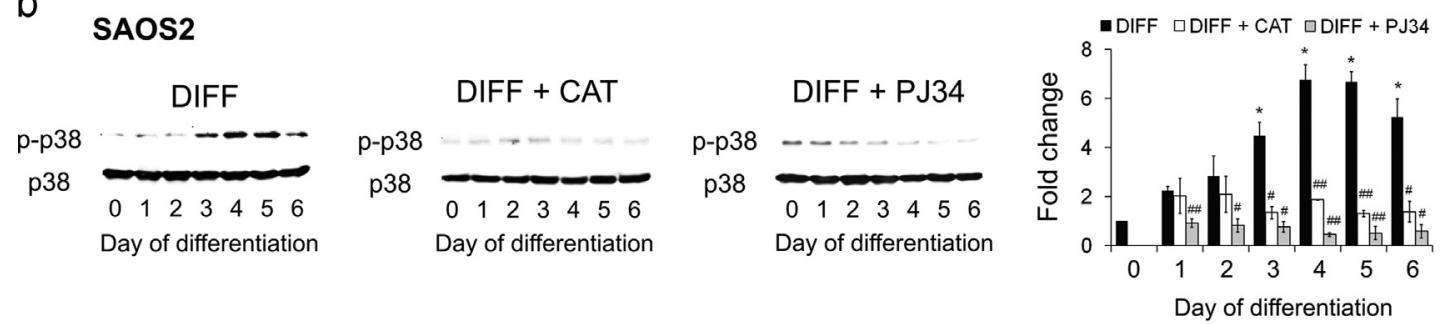

C

ShPARP1

CTRL

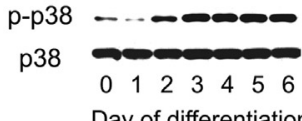

Day of differentiation

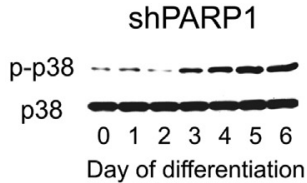

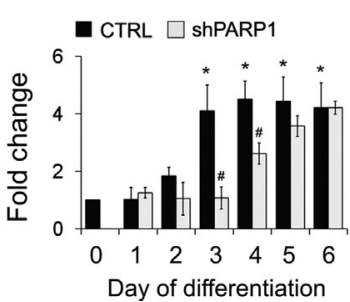

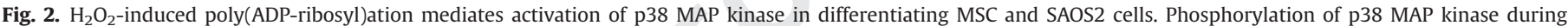

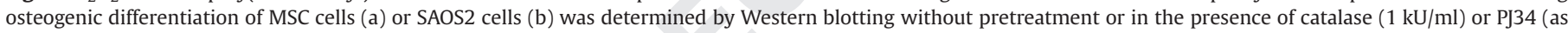

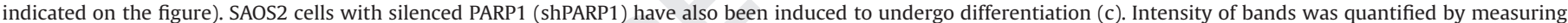

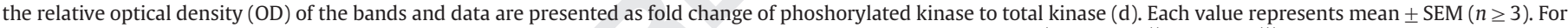

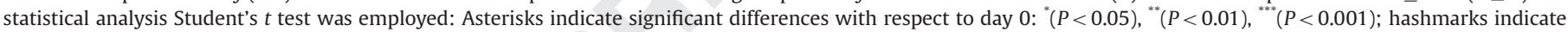

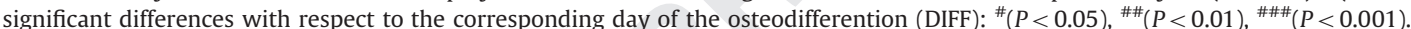

a downstream player in the hydrogen peroxide-PARP1 pathway contributing to cell death in differentiating MSC and SAOS-2 cells. Based on our previous observation that osteogenic differentiation and cell death appear to be closely coupled with each other we assumed that $\mathrm{p} 38$ should also inhibit the differentiation process. Indeed, the p38 inhibitor SB239063 suppressed markers of osteogenic differentiation such as calcium deposition (Fig. 4a, c, d, and f) and ALP activity (Fig. 4b and e) both in MSC and in SAOS-2 cultures. Moreover, experiments repeated with another p38 inhibitor (SB203580) confirmed the pivotal role of p38 in osteodifferentiation and cell death (data not shown). p38 inhibition had no effect on nondifferentiating cells that showed no sign of increased cell death.

Since these data clearly identified p38 as a downstream mediator of the $\mathrm{H}_{2} \mathrm{O}_{2}$-PARP1 differentiation and cell death pathway, in the following part of the study we focused on the characterization of the role of this MAP kinase in osteodifferentiation and coupled cell death.

The PARP1-p38 cytotoxic pathway culminates in cellular energetic failure during osteogenic differentiation-coupled cell death

Hydrogen peroxide-induced protein poly(ADP-ribosyl)ation prompted us to analyze cellular levels of NAD, the substrate of PARP1 which is also utilized as a cofactor in metabolic pathways, thereby linking PARP1 activity to metabolic failure, possibly contributing to cell death [11].

Osteogenic differentiation of SAOS-2 and MSC cells was accompanied by a progressive decrease in the concentration of intracellular
NAD (Fig. 5a and g). Supplementation of osteogenic medium with the PARP inhibitor PJ-34 $(1 \mu \mathrm{M})$ significantly protected cells against the loss of NAD (Fig. 5a and g) similar to PARP1 silencing (Fig. 5d). Moreover, nondifferentiating (day 0) shPARP1 cells demonstrated slightly but significantly higher values of total cellular NAD when compared to control cells (CTRL).

The loss of NAD was followed by the decrease in metabolic activity of differentiating cells. Intensity of glycolysis, mirrored by $\mathrm{ECAR}_{\mathrm{NM}}$ as well as ATP synthesis-associated mitochondrial respiration, mirrored by $\mathrm{OCR}_{\mathrm{BR}}$, declined as a consequence of osteogenic differentiation (Fig. 5). Simultaneous collapse of two major intracellular sources of ATP suggests metabolic failure possibly resulting from the NAD utilization by PARP1. In order to verify this hypothesis we differentiated both cell lines in the presence of PJ34 $(1 \mu \mathrm{M})$ and employed PARP1-silenced SAOS-2 cells (shPARP1 cells). Similar to PARP1 inhibition, PARP1 silencing also prevented deterioration of mitochondrial respiration and glycolysis, indicating the negative effects of this enzyme on cellular metabolism (Fig. 5).

To further characterize mitochondrial failure we tested the effects of mitochondrial uncoupling compounds (oligomycin, FCCP, and antimycin) in an extracellular flux assay in SAOS-2 and shPARP1 cells (Fig. S3) in order to determine respiratory reserve capacity $\left(\mathrm{OCR}_{\mathrm{RRC}}\right)$ and maximum respiration rate $\left(\mathrm{OCR}_{\mathrm{MRR}}\right)$. These two parameters provided further proof for restricted mitochondrial activity of differentiated cells and also confirmed protection by PARP1 silencing.

Based on the functional interplay between PARP1 and p38 and the role of PARP1 in differentiation-associated metabolic dysfunction, we set out to investigate the role of $\mathrm{p} 38$ in the metabolic alterations observed during osteodifferentiation. Supplementation 

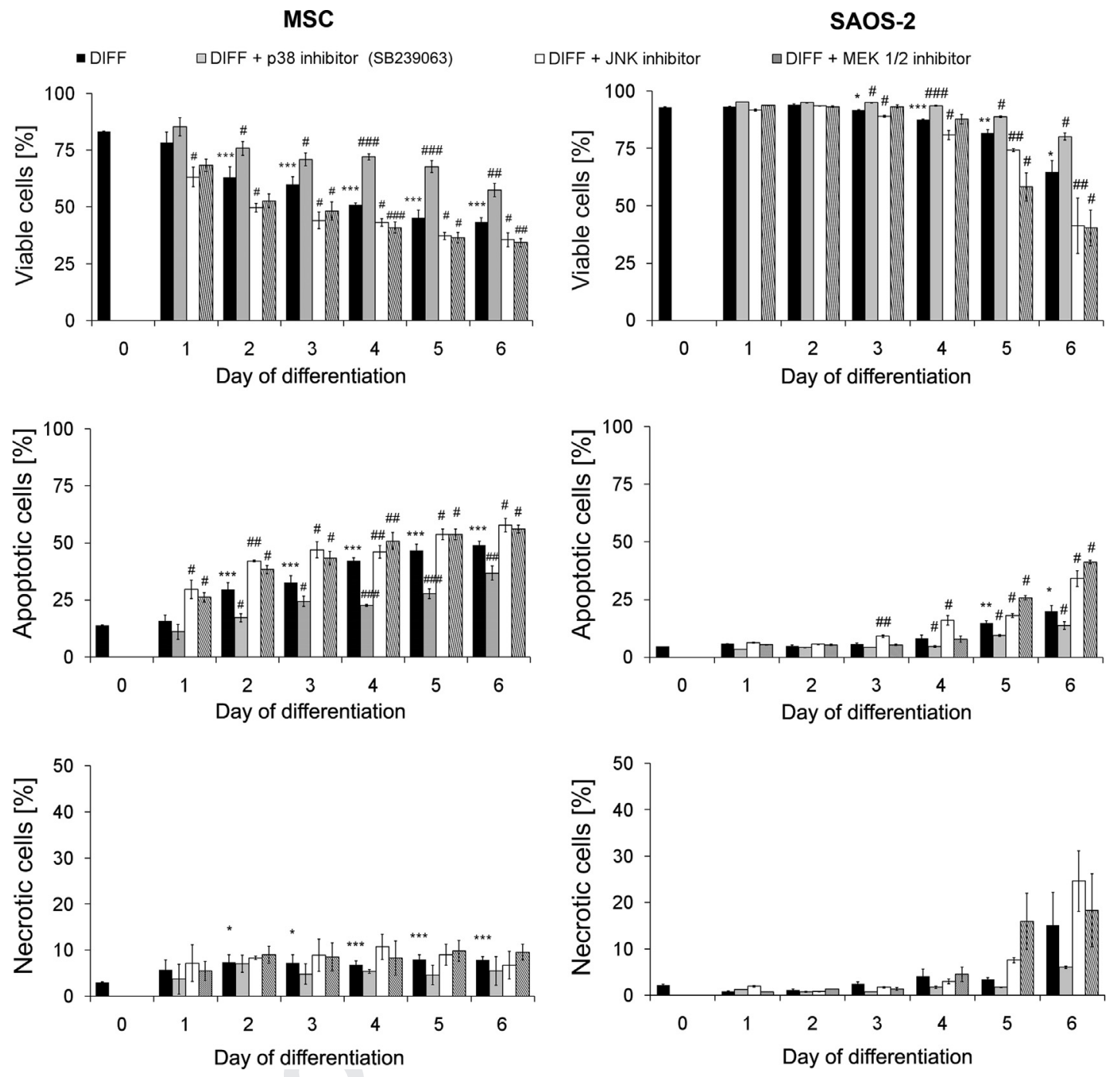

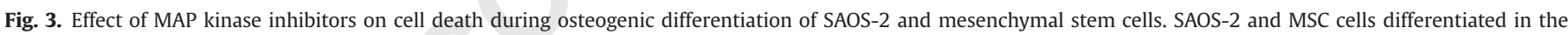

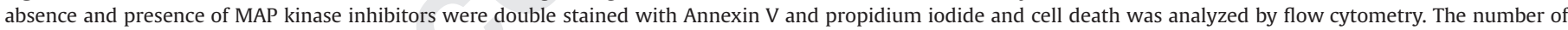

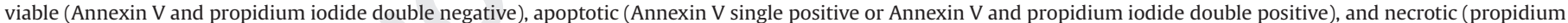

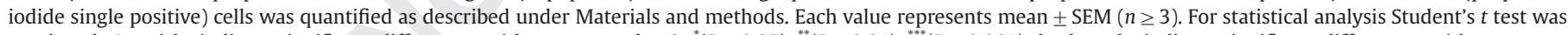

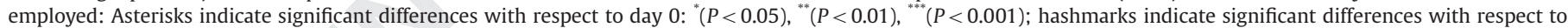

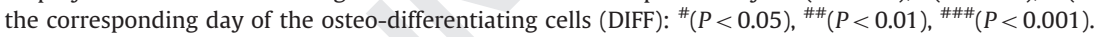

of osteogenic medium with the p38 inhibitor SB239063 for the whole differentiation process completely prevented the drop in the concentration of intracellular NAD in both studied cell types (Fig. 6a and d). Preservation of the cellular NAD pool was accompanied by preserved metabolic activity of differentiating cells (Fig. 6b, c, e, and f). However, protection of ATP synthesisassociated mitochondrial respiration $\left(\mathrm{OCR}_{\mathrm{BR}}\right)$ by the $\mathrm{p} 38$ inhibitor was more prevalent than that of glycolysis $\left(\mathrm{ECAR}_{\mathrm{NM}}\right)$ (Fig. 6b, c, e, and $\mathrm{f}$ ).

\section{PARP1 and p38 interact and colocalize with each other in differentiating cells}

Next we set out to investigate whether PARP1 and p38 interact with each other. We found that protein-protein interaction between the two proteins indeed develops during the differentiation process as evidenced by immunoprecipitation experiments (Fig. 7). Coimmunoprecipitation of p38 and PARP1 could be observed in two-way experiments: immunoprecipitation of PARP1 followed by Western blot detection of p38 (Fig. 7a) or immunoprecipitation of p38 or phospho-p38 followed by Western blot detection of PARP1 (Fig. 7b). Nonbinding (NB) controls (supernatants obtained after first centrifugation of beads) displayed virtually no positive bands (Fig. 7b). Parallel experiments performed without primary antibody also failed to immunoprecipitate any protein, indicating that binding is not due to nonspecific interaction between beads and our proteins of interest (data not shown). Immunofluorescent double staining for the two proteins revealed that in resting (nondifferentiating) cells p38 and PARP1 localize to the cytoplasm and to the nucleus, respectively. In differentiating cells, however, nuclear translocation of p38 can be observed and the two proteins colocalize in the nucleus (Fig. 7c).

\section{Discussion}

Regulation of osteogenic differentiation and cell death associated with the differentiation process is under the control of complex regulatory circuits. Recently we identified a redox regulatory pathway contributing to the control of osteodifferentiation and accompanying cell death [7]. A key event in this mechanism is 
MSC

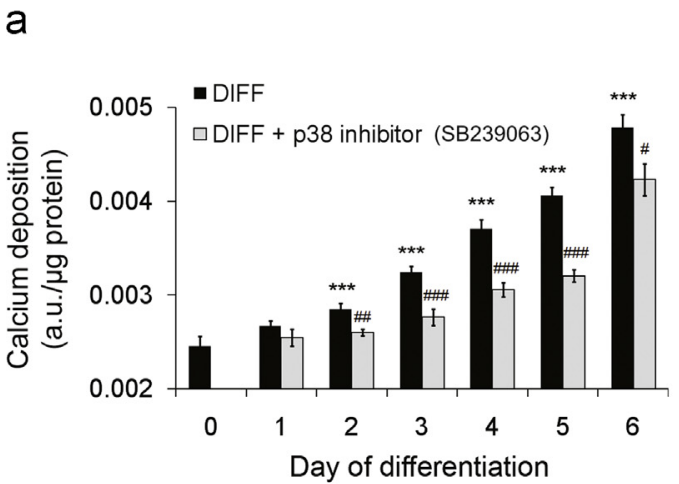

b

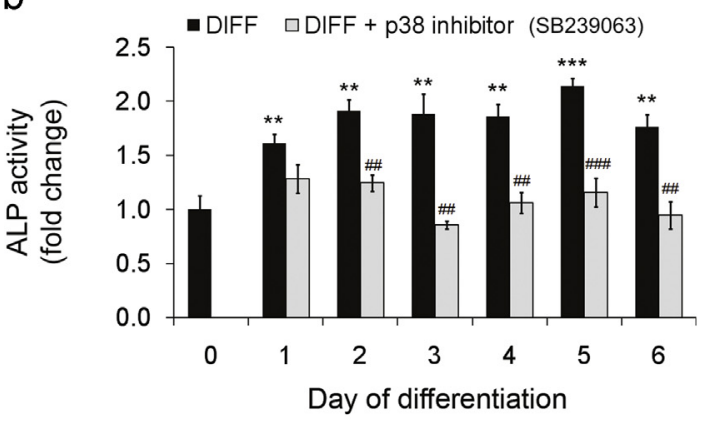

5th day
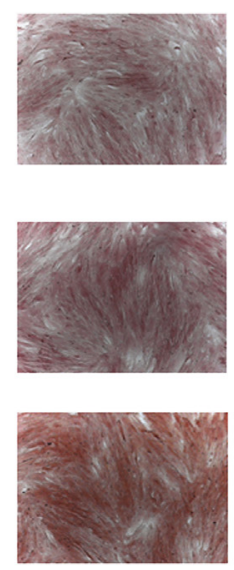

p38 inhibitor

(SB239063)

3rd day

4th day

\section{SAOS2}

d

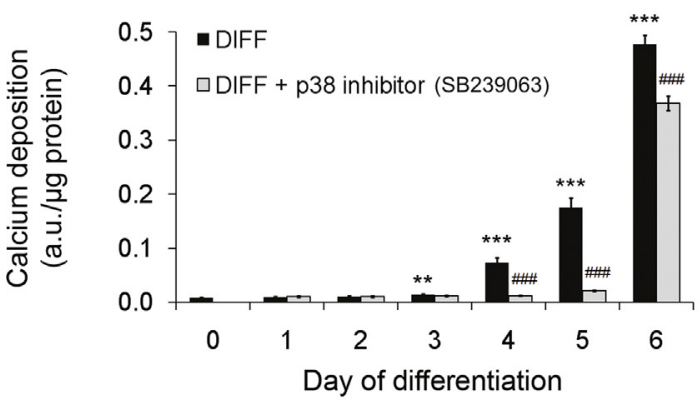

e

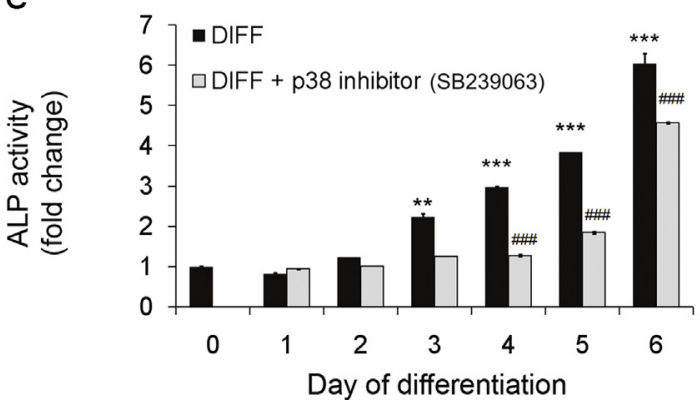

f

DIFF
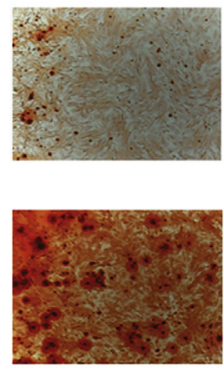

5th day
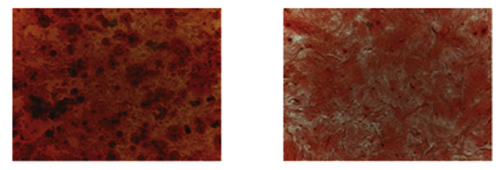

DIFF +

p38 inhibitor

(SB239063)
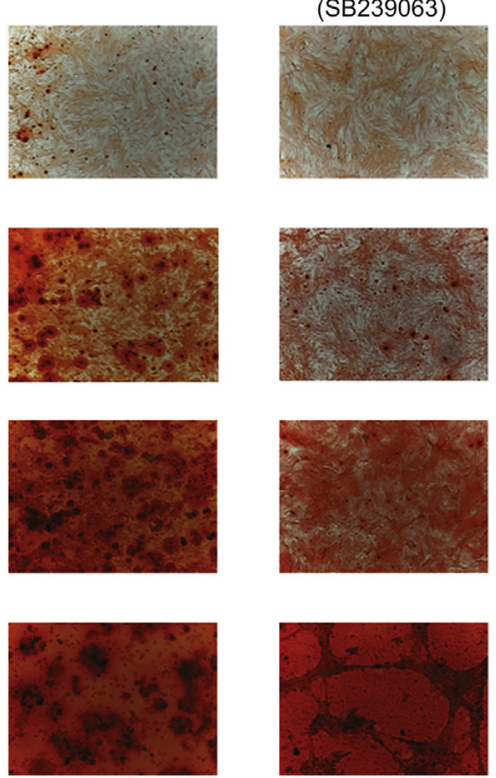

67

Fig. 4. p38 kinase mediates osteogenic differentiation of mesenchymal stem cells and SAOS-2 cells. The effect of the p38 inhibitor SB239063 (5 $\mu \mathrm{M})$ on osteogenic differentiation markers was studied during the course of osteogenic differentiation in both types of cells. Alkaline phosphatase activity (b and e) was determined in colorimetric assays (as described under Materials and methods). For evaluation of matrix mineralization cells were stained with Alizarin Red S (stains calcium). Alizarin Red $\mathrm{S}$-stained cultures were photographed with a microscope equipped with a digital camera (c and f) or Alizarin Red S was solubilized and absorbance was read at $540 \mathrm{~nm}$ (a and d). Each value represents mean $\pm \operatorname{SEM}(n \geq 3)$. For statistical analysis Student's $t$ test was employed: Asterisks indicate significant differences with respect to day 0 : ${ }^{*}(P<0.05),{ }^{* *}(P<0.01),{ }^{* * *}(P<0.001)$; hashmarks indicate significant differences with respect to the corresponding day of the osteodifferentiating cells $(\mathrm{DIFF}):{ }^{\#}(P<0.05)$, $\# \#(P<0.01), \# \# \#(P<0.001)$.

the activation of PARP1 in response to production of reactive oxygen species in differentiating cells. Analysis of mitochondrial superoxide production and NADPH oxidase (NOX) activity showed that superoxide may be released by both sources. However, MitoSOX oxidation occurred relatively late and thus might not be the primary source of ROS driving differentiation. The pattern of 


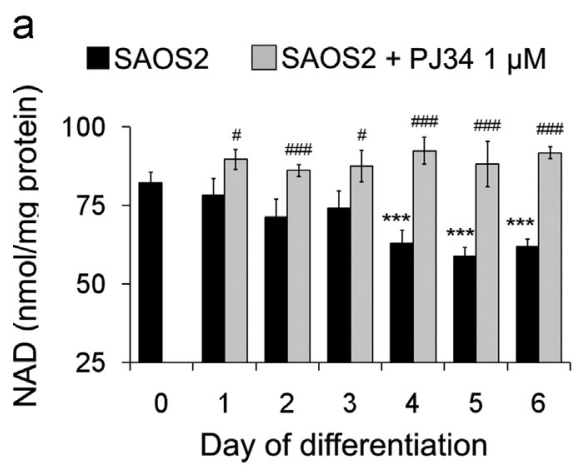

d

CTRL $\square$ ShPARP1

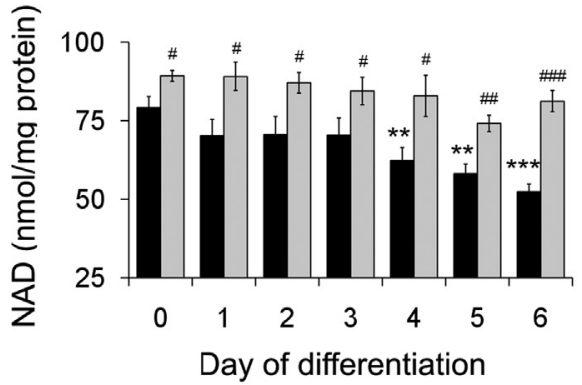

g

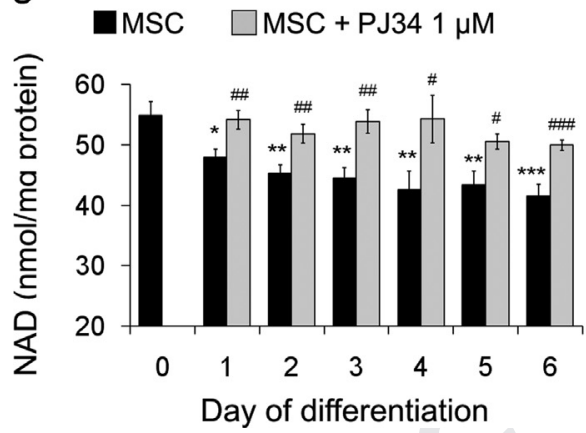

b

C

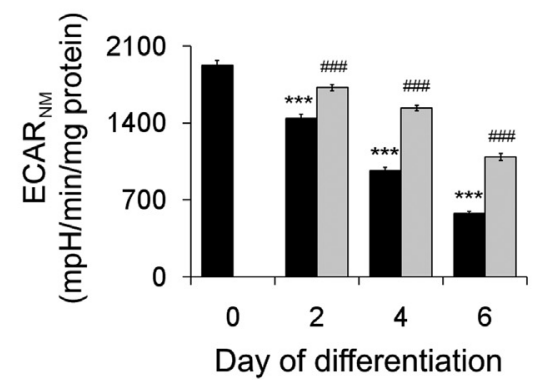

e

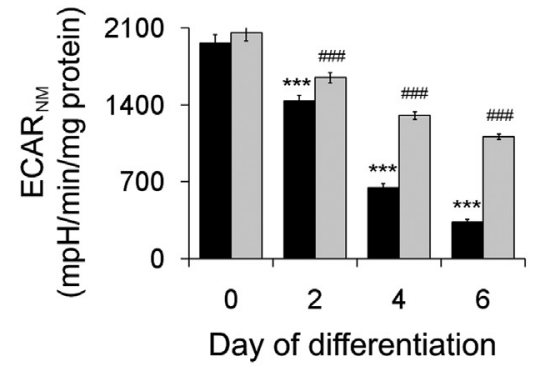

h

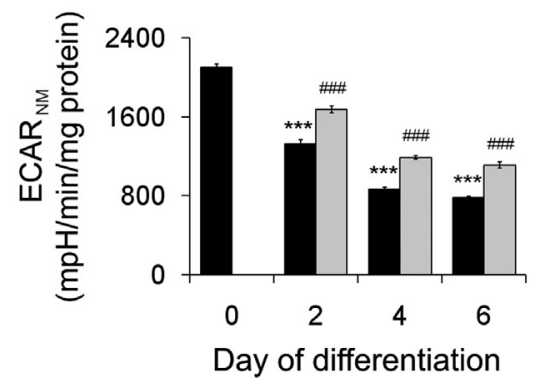

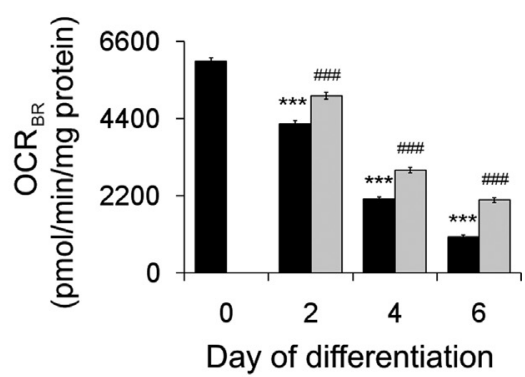

f

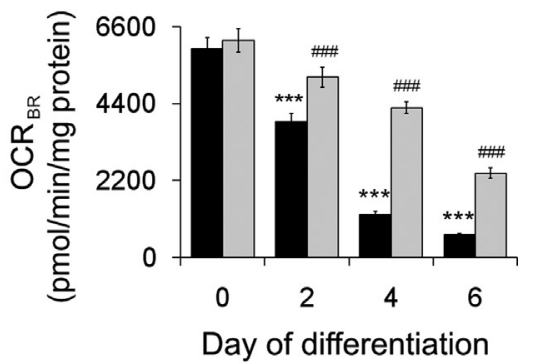

i

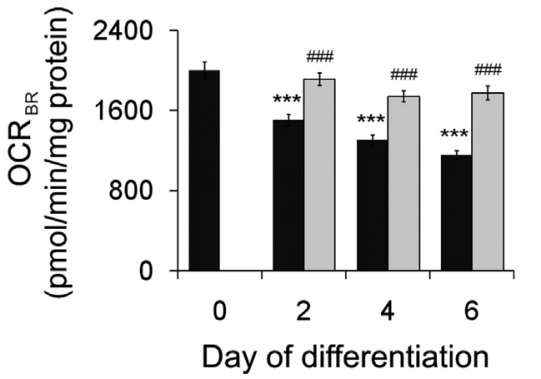

67

68

69

70

71

72

73

74

75

76

77

78

79

80

81

82

83

84

85

86

87

88

89

90

91

92

93

94

95

96

97

98

99

100

101

102

103

104

105

106

107

108

109

110

111

NOX activity (and expression of two NOX isoforms) closely resembled that of $\mathrm{H}_{2} \mathrm{O}_{2}$ production, suggesting that NOX may be the most likely source of $\mathrm{H}_{2} \mathrm{O}_{2}$ causing DNA damage and PARP activation in our model (Fig. S4). This is also in agreement with previous findings on the contribution of NOX to BMP2-stimulated osteoblast differentiation $[8,37]$. However, several open questions such as the nature of connection between ROS production and the activation of PARP1 as well as mediators of downstream signaling remain to be elucidated.

In cells exposed to relatively high concentrations of exogenous reactive oxygen species such as exogenous hydrogen peroxide or peroxynitrite, the trigger for PARP activation is ROS-induced DNA breakage [38]. However, several alternative mechanisms leading to the activation of PARP1 in the absence of DNA damage have also been described [39-42]. Here we have shown that a low concentration of hydrogen peroxide produced "physiologically" by cells induced to undergo osteodifferentiation is sufficient to induce DNA breakage as evidenced by the comet assay. DNA breaks are caused by endogenous hydrogen peroxide production as decomposition of hydrogen peroxide by catalase treatment prevented the formation of DNA breaks. The oxidative nature of DNA damage in this model was also indicated by the appearance of $80 \mathrm{HdG}$ in the differentiating cells. The finding of oxidative DNA damage in a differentiation model was slightly surprising to us in light of the generally held view that cells are abundantly equipped with a plethora of antioxidants (both enzymatic and nonenzymatic) to prevent significant macromolecular damage. It appears possible that extended production of low amounts of hydrogen peroxide can break through the barrier formed by antioxidant defense mechanisms and cause cellular damage. Similar observations have previously been made by Rodríguez-Vargas et al. [43] demonstrating ROS production, DNA damage, and PARP1 activation in cells undergoing starvation-induced autophagy, suggesting that this pathway may not be restricted to osteogenic differentiation but may be operational in various diverse cellular systems. In the autophagy model, PARP1 activation has been shown to positively feed back to ROS production. Activity of a similar vicious cycle cannot be excluded 


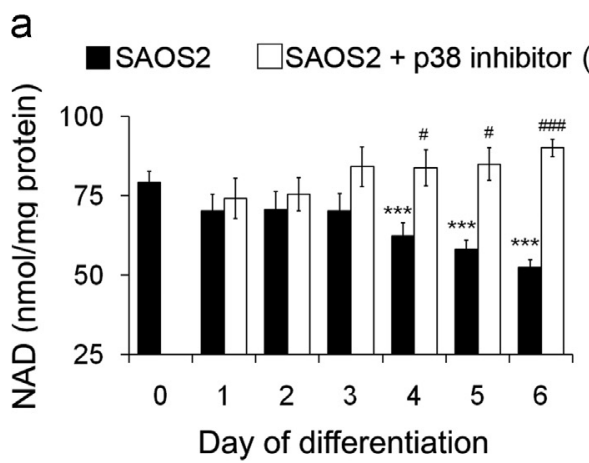

d

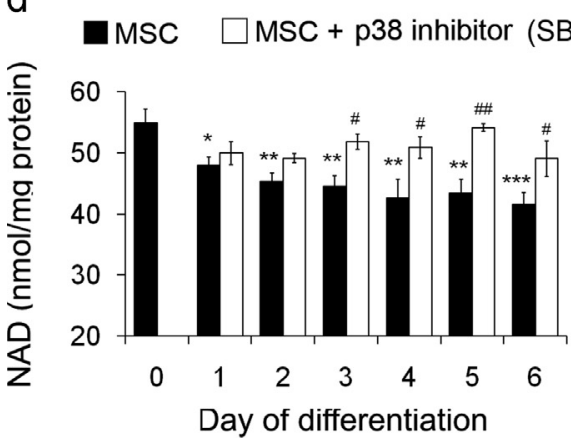

b

B239063)

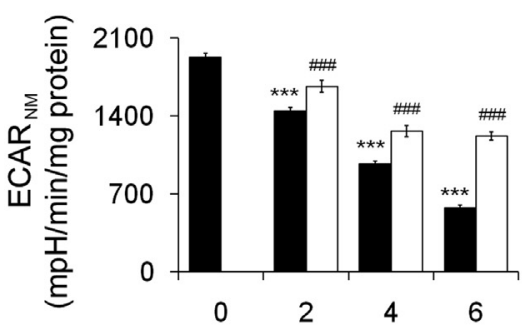

Day of differentiation
C

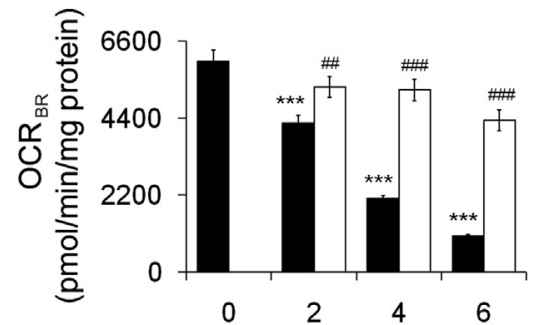

Day of differentiation f

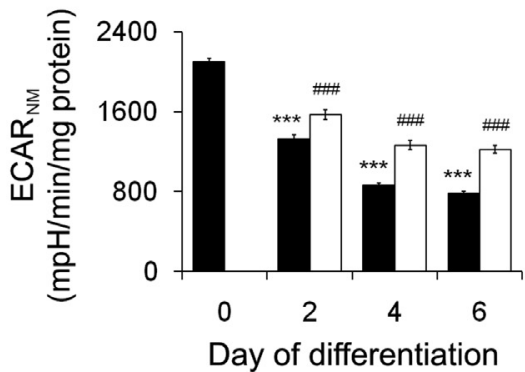

67

68

69

70

71

72

73

74

Fig. 6. p38 kinase contributes to metabolic collapse accompanying osteogenic differentiation. SAOS-2 (a-c) and mesenchymal stem cells (MSC) (d-f) were induced to

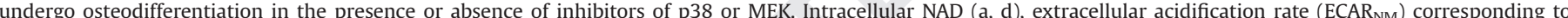
glycolytic activity (b, e), oxygen consumption rate $\left(\mathrm{OCR}_{\mathrm{BR}}\right)$ representing mitochondrial respiration (c, f) were determined with a Seahorse XF96 analyzer. Each bar represents mean + SEM $(n>3)$. For statistical analysis Student's $t$ test was employed: Asterisks indicate significant differences with respect to day $0:{ }^{*}(P<0.05)$, " $(P<0.01)$,

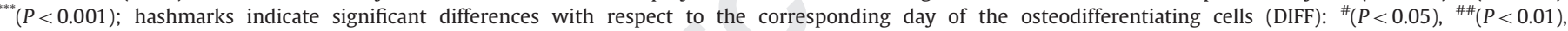
$\# \# \#(P<0.001)$. a

IP: $\alpha$-PARP1

Day of Differentiation

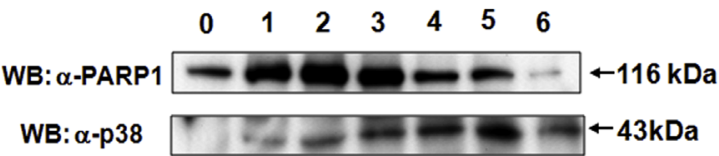

b
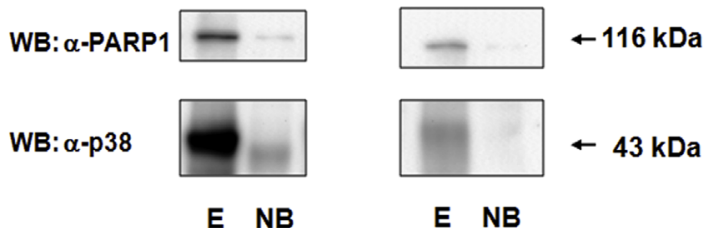

C
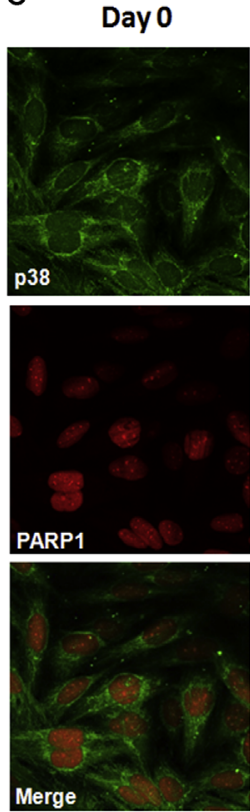

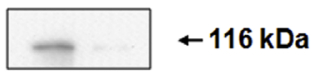

E NB
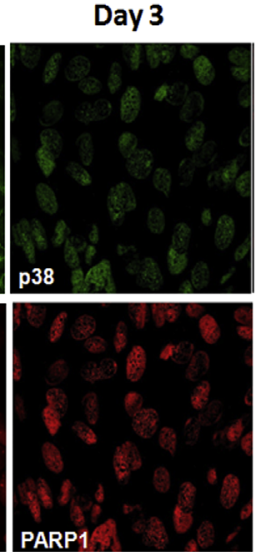

Fig. 7. Protein-protein interaction between and colocalization of PARP1 and p38 during osteogenic differentiation of SAOS-2 cells. Physical interaction between PARP1 and p38 was detected in immunoprecipitation experiments ( $a$ and b). Immunoprecipitation was carried out with anti-PARP1 (a), anti-p38 (b), or phospho-p38 (b) antibodies followed by Western blots for PARP1 and p38 (E, eluate; NB, nonbinding control). Colocalization of PARP1 and p38 as confirmed by double immunofluorescent staining is presented in panel (c). In contrast to undifferentiated samples (day 0), staining patterns observed on day 3 of differentiation show a clear colocalization of PARP1 and p38.

in osteodifferentiation, but confirming this hypothesis requires further investigation.

The central question in our current study was to identify mechanisms downstream of PARP1 which can mediate osteodifferentiationcoupled cell death. Activation of PARP1 can lead to cell death via different pathways: parthanatos, NAD/ATP depletion, and activation of death signaling routes such as the ones mediated by MAP kinases. The parthanatos pathway involves the release of the PAR polymer from the nucleus, its translocation to the mitochondria, and stimulation of the mitochondrial release of AIF. In turn AIF migrates to the nucleus where 
it triggers caspase-independent DNA fragmentation and necroptotic cell death. Previously we have demonstrated the lack of mitochondrio-nuclear translocation of AIF during osteodifferentiation-induced cell death [7]. Suppression of cellular metabolism (due to depletion of cellular NAD and consequently that of ATP) has also been made responsible for PARP-mediated cellular suicide. Here we found that osteogenic differentiation-coupled cell death is accompanied by depletion of NAD, the substrate of PARP, suppression of both glycolysis and basal as well as maximal mitochondrial respiration. Collapse of cellular energetics was due to ROS production as catalase prevented metabolic dysfunction of differentiating cells (data not shown). Moreover, activation of PARP1 is also a key mediator of differentiation-associated metabolic derailment as pretreatment of cells with the pharmacological PARP inhibitor PJ-34 or silencing PARP1 rescued cells from energy crisis. It seems, however, that the relationship between PARP activation and energetic collapse is more complex than simply being caused by NAD depletion as p38 MAP kinase was identified by this study as mediator of the energetic effects of PARylation (Fig. 6). MAP kinases have previously been linked to PARylation by several observations. However, the nature of this connection is not characterized in detail and displays clear modelspecific differences. In osteodifferentiation we found that all three MAP kinases display signs of activation. However, activation of JNK was not related to either hydrogen peroxide or PARP1. Furthermore, activation of Erk was only connected to PARylation but not to hydrogen peroxide. Nevertheless, p38 activation, was dependent both on hydrogen peroxide and on PARP1 and therefore in the rest of this study we focused on the role of p38 in osteodifferentiation. Based on previous studies reporting dephosphorylation of Erk by p38 [36] we have also investigated whether increased activation of Erk under PARP inhibition or PARP silencing is related to p38 but this mechanism could not be confirmed (data not shown). Using pharmacological inhibitors of the three MAP kinase pathways we have investigated the roles of p38, Erk1/2, and JNK in osteodifferentiation-coupled cell death. Similar to hydrogen peroxide decomposition by catalase or PARP inhibition/silencing, the inhibition of p38 but not that of the other two MAP kinases affected cell death in our model. Participation of p38 in redox regulation of cell death pathways is consistent with some other reports in the literature. For example Wei et al. [35] have shown that exogenous hydrogen peroxide activates p38 that mediates apoptosis of mesenchymal stem cells. Moreover, endogenous ROS production has also been shown to activate p38 in photodynamic cell death of cancer cell lines induced by a porphyrin derivative [44]. p38 signaling appears to converge on the mitochondria in our model as indicated by the partial prevention of mitochondrial dysfunction and glycolytic collapse in the presence of the p38 inhibitor. Similarly, Cai et al. [32] have found that in a model of sodium arsenite-induced apoptosis of PC12 cells, p38 phosphorylated the Bcl-2 family protein $\mathrm{Bim}_{\mathrm{EL}}$ and this proved to be essential for cell death. It seems plausible to hypothesize that in our model of osteodifferentiation, p38 may be activated by $\mathrm{H}_{2} \mathrm{O}_{2}$-PARP1 signaling and in turn, p38 may mediate mitochondrial dysfunction and consequent apoptosis. How far downstream from PARP1 activation p38 may function in this regulatory pathway was also investigated. We found that the interaction of these two proteins is rather direct as the two proteins interact with each other as verified by immunoprecipitation (Fig. 7). This interaction requires p38 to translocate from the cytoplasm to the nucleus. This translocation indeed takes place in differentiating cells and in the nucleus the two proteins closely colocalize (Fig. 7). The nuclear translocation of p38 has previously been reported in different stress states $[45,46]$ but not in any differentiation-related setting. The exact nature of the connection between PARP1 activation and p38 as well as the targets of p38 in the mitochondria and the nucleus requires further investigation.

Our current data provide further support for the closely coupled relationship between osteogenic differentiation and associated cell death. It appears that most of the regulators identified in our previous and current study affect both the differentiation pathway and the associated cell death. Hydrogen peroxide, PARP1, and p38 mediate both osteodifferentiation and related cell death. Dexamethason, used as inducer of differentiation in our model, also triggers both differentiation and apoptosis [47]. It appears plausible to hypothesize that apoptosis of a substantial number of osteoprogenitors is required for the efficient completion of the differentiation program and interventions affecting this death pathway will have the same type of effect on the differentiation process. In support of this hypothesis a recent report has shown that both necrotic and apoptotic cells of osteoblast lineage serve as nuclei for calcification on osteoblastic differentiation of mesenchymal stem cells [48].

In summary, we have established a new pathway promoting cell death in differentiating osteoprogenitor cells. The pathway involves production of hydrogen peroxide, DNA breakage, activation of PARP1, interaction between PARP1 and p38, and activation of p38 MAP kinase converging on metabolic dysfunction and culminating in cell death. These dead cells may serve as nuclei for calcification and osteogenic differentiation explaining the differentiation promoting effects of these events.

\section{Acknowledgments}

This research was supported by the European Union and the State of Hungary, cofinanced by the European Social Fund in the framework of TÁMOP 4.2.4.A/2-11/1-2012-0001 National Excellence Program. Direct costs of this study were supported by the Hungarian Science Research Fund (OTKA K82009, K112336, K108308). The authors declare no conflict of interest.

\section{Appendix A. Supplementary Information}

Supplementary data associated with this article can be found in the online version at http://dx.doi.org/10.1016/j.freeradbiomed. 2014.07.027.

\section{References}

[1] Shi, S.; Kirk, M.; Kahn, A. J. The role of type I collagen in the regulation of the osteoblast phenotype. J. Bone Miner. Res. 11:1139-1145; 1996.

[2] Clarke, B. Normal bone anatomy and physiology. Clin. J. Am. Soc. Nephrol 3 (Suppl 3):S131-139; 2008.

[3] Chen, G; Deng, C; TGF-beta, Li YP. and BMP signaling in osteoblast differentiation and bone formation. Int. J. Biol. Sci. 8(2):272-288; 2012.

[4] Jensen, ED; Gopalakrishnan, R; Westendorf, JJ. Regulation of gene expression in osteoblasts. Biofactors 36(1):25-32; 2010.

[5] Lian, JB; Javed, A; Zaidi, SK; Lengner, C; Montecino, M; van Wijnen, AJ; et al. Regulatory controls for osteoblast growth and differentiation: role of Runx/ Cbfa/AML factors. Crit. Rev. Eukaryot. Gene Expr. 14(1-2):1-41; 2004.

[6] Javed, A; Chen, H; Ghori, FY. Genetic and transcriptional control of bone formation. Oral Maxillofac. Surg. Clin. North Am. 22(3):283-293; 2010. (v).

[7] Robaszkiewicz, A; Erdelyi, K; Kovacs, K; Kovacs, I; Bai, P; Rajnavolgyi, E; et al Hydrogen peroxide-induced poly(ADP-ribosyl)ation regulates osteogenic differentiation-associated cell death. Free Radic. Biol. Med. 53(8):1552-1564; 2012.

[8] Mandal, CC; Ganapathy, S; Gorin, Y; Mahadev, K; Block, K; Abboud, HE; et al. Reactive oxygen species derived from Nox4 mediate BMP2 gene transcription and osteoblast differentiation. Biochem. J. 433(2):393-402; 2011.

[9] Arakaki, N; Yamashita, A; Niimi, S; Yamazaki, T. Involvement of reactive oxygen species in osteoblastic differentiation of MC3T3-E1 cells accompanied by mitochondrial morphological dynamics. Biomed. Res. 34(3):161-166; 2013.

[10] Schreiber, V; Dantzer, F; Ame, JC de Murcia G. Poly(ADP-ribose): nove functions for an old molecule. Nat. Rev. Mol. Cell. Biol. 7(7):517-528; 2006.

[11] Virag L, Robaszkiewicz A, Vargas JM, Javier Oliver F. Poly(ADP-ribose) signaling in cell death. Mol. Aspects Med. 2013. 10.1016/j.mam.2013.01.007.

[12] Dantzer, F; Santoro, R. The expanding role of PARPs in the establishment and maintenance of heterochromatin. FEBS J. 280(15):3508-3518; 2013.

[13] Kraus, WL; Hottiger, MO. PARP1 and gene regulation: Progress and puzzles. Mol. Aspects Med. ; 2013. http://dx.doi.org/10.1016/j.mam.2013.01.005.

[14] Espejel, S; Klatt, P; Ménissier-de Murcia, J; Martín-Caballero, J; Flores, JM; Taccioli, G; et al. Impact of telomerase ablation on organismal viability, aging, 
and tumorigenesis in mice lacking the DNA repair proteins PARP-1, Ku86, or DNA-PKcs. J. Cell Biol. 167(4):627-638; 2004.

[15] Bai, P; Canto, C. The role of PARP1 and PARP-2 enzymes in metabolic regulation and disease. Cell Metab. 16(3):290-295; 2012.

[16] Burkle, A; Virag, L. Poly(ADP-ribose): PARadigms and PARadoxes. Mol. Aspects Med. ; 2013. http://dx.doi.org/10.1016/i.mam.2012.12.010.

[17] Berger, NA; Berger, SJ; Catino, DM; Petzold, SJ; Robins, RK. Modulation of nicotinamide adenine dinucleotide and poly(adenosine diphosphoribose) metabolism by the synthetic " $\mathrm{C}$ " nucleoside analogs, tiazofurin and selenazofurin. A new strategy for cancer chemotherapy. J. Clin. Invest. 75(2):702-709; 1985.

[18] Yu, SW; Wang, H; Poitras, MF; Coombs, C; Bowers, WJ; Federoff, HJ; et al. Mediation of poly(ADP-ribose) polymerase-1-dependent cell death by apoptosis-inducing factor. Science 297(5579):259-263; 2002.

[19] Szanto, A; Hellebrand, EE; Bognar, Z; Tucsek, Z; Szabo, A; Gallyas Jr. F; et al. PARP1 inhibition-induced activation of PI-3-kinase-Akt pathway promotes resistance to taxol. Biochem. Pharmacol. 77(8):1348-1357; 2009.

[20] Xu, Y; Huang, S; Liu, ZG; Han, J. Poly(ADP-ribose) polymerase-1 signaling to mitochondria in necrotic cell death requires RIP1/TRAF2-mediated JNK1 activation. J. Biol. Chem. 281(13):8788-8795; 2006.

[21] Ethier, C; Labelle, Y; Poirier, GG. PARP1-induced cell death through inhibition of the MEK/ERK pathway in MNNG-treated HeLa cells. Apoptosis 12 (11):2037-2049; 2007.

[22] Kauppinen, TM; Chan, WY; Suh, SW; Wiggins, AK; Huang, EJ; Swanson, RA. Direct phosphorylation and regulation of poly(ADP-ribose) polymerase-1 by extracellular signal-regulated kinases 1/2. Proc. Natl. Acad. Sci. USA 103 (18):7136-7141; 2006

[23] Galbis-Martinez, M; Saenz, L; Ramirez, P; Parrilla, P; Yelamos, J. Poly(ADPribose) polymerase-1 modulates interferon-gamma-inducible protein (IP)-10 expression in murine embryonic fibroblasts by stabilizing IP-10 mRNA. Mol. Immunol. 47(7-8):1492-1499; 2010.

[24] Racz, B; Hanto, K; Tapodi, A; Solti, I; Kalman, N; Jakus, P; et al. Regulation of MKP-1 expression and MAPK activation by PARP1 in oxidative stress: a new mechanism for the cytoplasmic effect of PARP1 activation. Free Radic. Biol. Med. 49(12):1978-1988; 2010.

[25] Erdelyi, K; Bai, P; Kovacs, I; Szabo, E; Mocsar, G; Kakuk, A; et al. Dual role of poly(ADP-ribose) glycohydrolase in the regulation of cell death in oxidatively stressed A549 cells. FASEB J. 23(10):3553-3563; 2009.

[26] Kovacs, K; Erdelyi, K; Hegedus, C; Lakatos, P; Regdon, Z; Bai, P; et al. Poly(ADPribosyl)ation is a survival mechanism in cigarette smoke-induced and hydrogen peroxide-mediated cell death. Free Radic. Biol. Med. 53(9):1680-1688; 2012.

[27] Rothgiesser, KM; Erener, S; Waibel, S; Lüscher, B; Hottiger, MO. SIRT2 regulates NF- $\mathrm{KB}$ dependent gene expression through deacetylation of $\mathrm{p} 65$ Lys310. J. Cell Sci. 123(Pt 24):4251-4258; 2010.

[28] Modis, K; Gero, D; Erdelyi, K; Szoleczky, P; DeWitt, D; Szabo, C. Cellular bioenergetics is regulated by PARP1 under resting conditions and during oxidative stress. Biochem. Pharmacol. 83(5):633-643; 2012.

[29] Yamamoto, A; Nakamura, Y; Kobayashi, N; Iwamoto, T; Yoshioka, A; Kuniyasu, H; et al. Neurons and astrocytes exhibit lower activities of global genome nucleotide excision repair than do fibroblasts. DNA Repair (Amst.) 6(5):649-657; 2007.

[30] Wang, X; Son, YO; Chang, Q; Sun, L; Hitron, JA; Budhraja, A; et al. NADPH oxidase activation is required in reactive oxygen species generation and cell transformation induced by hexavalent chromium. Toxicol. Sci. 123(2):399-410; 2011.

[31] Robaszkiewicz, A; Bartosz, G; Pitt, AR; Thakker, A; Armstrong, RA; Spickett, CM; et al. HOCl-modified phosphatidylcholines induce apoptosis and redox imbalance in HUVEC-ST cells. Arch. Biochem. Biophys. 548:1-10; 2014.
[32] Cai, B; Chang, SH; Becker, EB; Bonni, A; Xia, Z. p38 MAP kinase mediates apoptosis through phosphorylation of BimEL at Ser-65. J. Biol. Chem. 281 (35):25215-25222; 2006

[33] Chang, L; Karin, M. Mammalian MAP kinase signaling cascades. Nature 410 (6824):37-40; 2001.

[34] Lu, Z; Xu, S.; ERK1/2, MAP kinases in cell survival and apoptosis. IUBMB Life 58 (11):621-631; 2006.

[35] Wei, H; Li, Z; Hu, S; Chen, X; Cong, X. Apoptosis of mesenchymal stem cells induced by hydrogen peroxide concerns both endoplasmic reticulum stress and mitochondrial death pathway through regulation of caspases, p38 and JNK. J. Cell Biochem. 111(4):967-978; 2010.

[36] Li, SP; Junttila, MR; Han, J; Kahari, VM; Westermarck, J. p38 Mitogen-activated protein kinase pathway suppresses cell survival by inducing dephosphorylation of mitogen-activated protein/extracellular signal-regulated kinase kinase1,2. Cancer Res. 63(13):3473-3477; 2003.

[37] Liberman, M; Johnson, RC; Handy, DE; Loscalzo, J; Leopold, JA. Bone morphogenetic protein-2 activates NADPH oxidase to increase endoplasmic reticulum stress and human coronary artery smooth muscle cell calcification. Biochem. Biophys. Res. Commun. 413(3):436-441; 2011.

[38] Virag, L; Szabo, C. The therapeutic potential of poly(ADP-ribose) polymerase inhibitors. Pharmacol. Rev. 54(3):375-429; 2002.

[39] Ju, BG; Solum, D; Song, EJ; Lee, KJ; Rose, DW; Glass, CK; et al. Activating the PARP1 sensor component of the groucho/ TLE1 corepressor complex mediates a CaMKinase IIdelta-dependent neurogenic gene activation pathway. Cell 119 (6):815-829; 2004.

[40] Potaman, VN; Shlyakhtenko, LS; Oussatcheva, EA; Lyubchenko, YL; Soldatenkov, VA. Specific binding of poly(ADP-ribose) polymerase-1 to cruciform hairpins. J. Mol. Biol. 348(3):609-615; 2005.

[41] Clark, NJ; Kramer, M; Muthurajan, UM; Luger, K. Alternative modes of binding of poly(ADP-ribose) polymerase 1 to free DNA and nucleosomes. J. Biol. Chem. 287(39):32430-32439; 2012.

[42] Cohen-Armon, M; Visochek, L; Rozensal, D; Kalal, A; Geistrikh, I; Klein, R; et al. DNA-independent PARP1 activation by phosphorylated ERK2 increases Elk1 activity: a link to histone acetylation. Mol. Cell 25(2):297-308; 2007.

[43] Rodriguez-Vargas, JM; Ruiz-Magana, MJ; Ruiz-Ruiz, C; Majuelos-Melguizo, J: Peralta-Leal, A; Rodriguez, MI; et al. ROS-induced DNA damage and PARP1 are required for optimal induction of starvation-induced autophagy. Cell Res. 22 (7):1181-1198; 2012.

[44] Kralova, J; Dvorak, M; Koc, M; Kral, V. p38 MAPK plays an essential role in apoptosis induced by photoactivation of a novel ethylene glycol porphyrin derivative. Oncogene 27(21):3010-3020; 2008.

[45] Gong, X; Ming, X; Deng, P; Jiang, Y. Mechanisms regulating the nuclear translocation of p38 MAP kinase. J. Cell Biochem. 110(6):1420-1429; 2010.

[46] Mertenskötter, A; Keshet, A; Gerke, P; Paul, RJ. The p38 MAPK PMK-1 shows heat-induced nuclear translocation, supports chaperone expression, and affects the heat tolerance of Caenorhabditis elegans. Cell Stress Chaperones 18(3):293-306; 2013

[47] Kim, SM; Kim, YG; Park, JW; Lee, JM; Suh, JY. The effects of dexamethasone on the apoptosis and osteogenic differentiation of human periodontal ligament cells. J. Periodontal Implant Sci 43(4):168-176; 2013.

[48] Fujita H, Yamamoto M, Ogino T, Kobuchi H, Ohmoto N, Aoyama E, et al. Necrotic and apoptotic cells serve as nuclei for calcification on osteoblastic differentiation of human mesenchymal stem cells in vitro. Cell Biochem. Funct. 2013. $10.1002 /$ cbf.2974. 